\title{
Tratamento endovascular do aneurisma da aorta abdominal infrarrenal em pacientes com anatomia favorável para o procedimento - experiência inicial em um serviço universitário
}

\section{Endovascular treatment of infrarenal abdominal aortic aneurysm in patients with favorable anatomy for the repair - initial experience in a university hospital}

José Manoel da Silva Silvestre', Fernando Motta², Wander Eduardo Sardinha³, Domingos de Morais Filho ${ }^{4}$, Fernando Thomazinho5, Guilherme da Silva Silvestre ${ }^{6}$, Igor Schincariol Perozin ${ }^{7}$

\begin{abstract}
Resumo
Contexto: Desde sua introdução, em 1991, o reparo endovascular do aneurisma da aorta abdominal infrarrenal tem se tornado uma alternativa atraente para o tratamento dessa doença. Avaliar nossos resultados iniciais quanto à segurança e eficácia dessa técnica nos levou à realização deste estudo. Objetivos: Analisar a mortalidade perioperatória, a sobrevida tardia, as reoperações, as taxas de perviedade e o comportamento do saco aneurismático em pacientes com anatomia favorável para a realização do procedimento.

Métodos: Trata-se de um estudo longitudinal, observacional e retrospectivo realizado entre outubro de 2004 e janeiro de 2009 com 41 pacientes que foram submetidos à correção endovascular do aneurisma de aorta abdominal infrarrenal por apresentarem anatomia favorável para o procedimento. Foram analisados os achados dos exames diagnósticos, o tratamento e o seguimento em todos os pacientes.

Resultados: Foram implantadas, com sucesso, 31 (75,6\%) próteses bifurcadas e 10 (24,5\%) monoilíacas, de 5 diferentes marcas. O diâmetro médio dos aneurismas fusiformes era de $62 \mathrm{~mm}$. A mortalidade perioperatória foi de 4,8\% e a sobrevida tardia, 90,2\%. Durante o acompanhamento médio de 30 meses, 2 (4,8\%) pacientes necessitaram de reintervenção, um por migração da endoprótese e outro por vazamento tipo II. Dois (4,8\%) pacientes apresentaram oclusão de ramo da prótese. Oito (19,5\%) vazamentos foram diagnosticados e não houve nenhuma rotura dos aneurismas.

Conclusão: Apesar do pequeno número de pacientes, os resultados observados parecem justificar a realização do procedimento endovascular nos pacientes com anatomia favorável.
\end{abstract}

Palavras-chave: Aneurisma da aorta abdominal; prótese vascular; arteriosclerose.

\begin{abstract}
Background: Since its introduction in 1991, endovascular repair of infrarenal aortic aneurysms has become an attractive option to treat this disease. The evaluation of our initial results about safety and efficacy of this technique has led us to carry out this study.

Objectives: To analyze perioperative mortality, late survival, reoperations, patency rates and the aneurysmal sac behavior in patients with favorable anatomy for this procedure.

Methods: A longitudinal, observational and retrospective study was conducted from October 2004 to January 2009 , involving 41 patients with favorable anatomy for endovascular repair of infrarenal aortic aneurysm. The findings of diagnostic exams, the treatment and follow-up results were analyzed.
\end{abstract}

Trabalho realizado na Disciplina de Angiologia e Cirurgia Vascular do Departamento de Clínica Cirúrgica da Universidade Estadual de Londrina.

Trabalho apresentado no XI Encontro Paranaense de Cirurgia Vascular, Curitiba (PR) - Novembro de 2009.

${ }^{1}$ Doutor; Professor Associado da Disciplina de Angiologia e Cirurgia Vascular do Departamento de Clínica Cirúrgica da Universidade Estadual de Londrina (UEL). Responsável pela Angiorradiologia e Cirurgia Endovascular do Hospital Universitário Regional do Norte do Paraná, Londrina (PR), Brasil.

${ }^{2}$ Residente de Angiorradiologia e Cirurgia Endovascular do Hospital Universitário Regional do Norte do Paraná, Londrina (PR), Brasil.

${ }^{3}$ Doutor; Professor Adjunto. Chefe da Disciplina de Angiologia e Cirurgia Vascular do Departamento de Clínica Cirúrgica da Universidade Estadual de Londrina (UEL), Londrina (PR), Brasil.

${ }^{4}$ Doutor; Professor Adjunto da Disciplina de Angiologia e Cirurgia Vascular do Departamento de Clínica Cirúrgica da Universidade Estadual de Londrina (UEL), Londrina (PR), Brasil.

${ }^{5}$ Ex-residente de Angiorradiologia e Cirurgia Endovascular do Hospital Universitário Regional do Norte do Paraná, Londrina (PR), Brasil.

${ }^{6}$ Acadêmico de Medicina da Universidade Estadual do Oeste do Paraná (UNIOESTE), Cascavel (PR), Brasil.

${ }^{7}$ Acadêmico de Medicina da Universidade Estadual de Londrina (UEL), Londrina (PR), Brasil.

Não foram declarados conflitos de interesse associados à publicação deste artigo.

Submetido em: 04.04.10. Aceito em: 04.09.10

J Vasc Bras. 2011;10(1):31-39. 
Results: Thirty-one (75.6\%) bifurcated and 10 (24.5\%) mono-iliac prosthesis of five different brands were successfully implanted. The average diameter of the fusiform aneurysms was $62 \mathrm{~mm}$. Perioperative mortality rate was $4.8 \%$ and late survival was $90.2 \%$. During the mean follow-up period of 30 months, two (4.8\%) patients needed re-intervention, one for migration of the endoprosthesis and the other for type II endoleak. Two (4.8\%) patients presented occlusion of an endograft branch. Eight (19.5\%) endoleaks were diagnosed and there was no aneurysm rupture.

Conclusion: Despite the small number of patients, the results seem to justify the performance of endovascular therapy in patients with favorable anatomy.

Keywords: Aortic aneurysm, abdominal; blood vessel prosthesis; arteriosclerosis.

\section{Introdução}

Os aneurismas da aorta abdominal infrarrenal (AAA) ocorrem em 5\% dos pacientes acima de 65 anos. Sua ruptura é fatal em 80 a $90 \%$ dos casos quando as mortes préhospitalares são incluídas. O reparo cirúrgico aberto é considerado efetivo, com taxa de falência do enxerto de apenas $0,3 \%$ ao ano. No entanto, possui taxas de morbimortalidade não desprezíveis, com período de recuperação que pode chegar a vários meses ${ }^{1-3}$.

Muitos pacientes portadores de AAA apresentam comorbidades que os tornam de alto risco para a correção dessa anormalidade. Com o desenvolvimento, a partir do relato de Parodi ${ }^{4}$, da técnica endovascular para a correção dos AAA nesse grupo de pacientes, esse tipo de reparo foi ampliando suas indicações, e mesmo pacientes com risco cirúrgico proibitivo passaram a ter seus aneurismas corrigidos pelo reparo endovascular, desde que apresentassem condições anatômicas favoráveis para sua realização.

O objetivo do presente trabalho foi descrever a nossa experiência inicial com a correção endovascular do AAA em pacientes com anatomia adequada para esse tipo de reparo, relatando a mortalidade perioperatória, a sobrevida tardia, as reintervenções, as taxas de perviedade e o comportamento do saco aneurismático.

Tabela 1 - Comorbidades apresentadas pelos pacientes

\begin{tabular}{lc}
\hline Comorbidades & Número (\%) \\
\hline Hipertensão arterial & $25(61,0)$ \\
Tabagismo & $22(53,7)$ \\
Dislipidemia & $17(41,5)$ \\
Doença arterial coronariana & $13(31,7)$ \\
Insuficiência cardíaca congestiva & $11(26,8)$ \\
Doença pulmonar obstrutiva crônica & $9(22,0)$ \\
Diabetes mellitus & $6(14,6)$ \\
Doença arterial obstrutiva periférica & $4(9,8)$ \\
Insuficiência renal crônica & $3(7,8)$ \\
Estenose de artéria renal & $1(2,4)$ \\
Trombocitemia & $1(2,4)$ \\
\hline
\end{tabular}

\section{Casuística e método}

Entre outubro de 2004 até janeiro de 2009, 41 pacientes foram submetidos ao reparo endovascular do AAA. Entre as comorbidades apresentadas pelos pacientes, a hipertensão arterial e o tabagismo foram as mais comuns, seguidas pela dislipidemia, doença arterial coronariana, insuficiência cardíaca e doença pulmonar obstrutiva crônica. A frequência das comorbidades pode ser vista na Tabela 1. Este estudo foi aprovado pelo Comitê de Ética em Pesquisa Envolvendo Seres Humanos, do Hospital Universitário Regional, do Norte do Paraná.

\section{Seleção dos pacientes}

Foram considerados como indicação para correção os pacientes do sexo masculino que apresentassem aneurismas fusiformes com diâmetro maior que $55 \mathrm{~mm}$, e do sexo feminino, maiores que $50 \mathrm{~mm}$. Os aneurismas saculares foram corrigidos independentemente de seu diâmetro. De acordo com a classificação da Sociedade Americana de Anestesiologia, $16(39,0 \%)$ pacientes foram classificados como ASA II, $22(53,6 \%)$ como ASA III e apenas $3(7,3 \%)$ como ASA IV; porém, essa estratificação de risco cirúrgico não foi analisada como critério de inclusão e/ou exclusão.

O procedimento foi planejado baseado em angiotomografia abdominal com contraste e reconstrução tridimensional e selecionado de acordo com os seguintes critérios: colo proximal com extensão igual ou superior a $15 \mathrm{~mm}$, angulação desse colo inferior a $60^{\circ}$, diâmetro das artérias ilíacas externas igual ou superior a $7 \mathrm{~mm}$, presença de trombos e/ou calcificação inferiores a 50\% da circunferência do colo proximal (Tabela 2). Aqueles que preencheram esses pré-requisitos foram considerados como tendo anatomia favorável e foram encaminhados para o tratamento endovascular de forma consecutiva. O resultado da avaliação do risco cirúrgico cardiológico e/ou anestésico não foi considerado na inclusão e/ou exclusão dos pacientes. 


\section{Técnica do procedimento endovascular}

Os pacientes foram tratados no setor de hemodinâmica do Hospital Universitário Regional do Norte do Paraná e no Hospital do Coração de Londrina.

Os procedimentos foram realizados sob anestesia geral e/ou bloqueio (ráqui/peridural). A profilaxia antimicrobiana foi realizada conforme orientação da Comissão de Controle de Infecção Hospitalar, sendo empregada a vancomicina por via endovenosa na dose única de $1,0 \mathrm{~g}$ aplicada no momento da indução anestésica. A abordagem preferencial foi da artéria femoral comum. Na impossibilidade desta, era realizada abordagem da artéria ilíaca externa por acesso extraperitoneal. O controle fluoroscópico foi feito com aparelho Siemens ${ }^{\oplus}$. Foram utilizados os dispositivos Apolo $^{\circledR}$, Excluder ${ }^{\oplus}$, Talent ${ }^{\oplus}$, Ella ${ }^{\oplus}$ e Aorfix ${ }^{\oplus}$. O pós-operatório foi conduzido, inicialmente, em Unidade de Terapia Intensiva (UTI).

\section{Acompanhamento pós-operatório}

Os pacientes foram acompanhados com avaliação após 30 dias, 6 meses e 12 meses do procedimento. Após o primeiro ano, as consultas foram realizadas a cada 12 meses. Em cada consulta foram realizados ecoDoppler, e/ou angiotomografia. Nos casos em que o ecoDoppler detectava crescimento do aneurisma e/ou vazamentos, realizávamos angiotomografia. Para investigação das possíveis fraturas da endoprótese, complementávamos o estudo com o raio $\mathrm{X}$ simples. O tempo de acompanhamento variou de 7 a 58 meses (mediana de 30 meses). Nenhum paciente deixou de ser acompanhado e avaliado.

\section{Análise estatística}

A análise estatística foi realizada usando o programa Stat-Graphics, versão 5.1. As variáveis foram relatadas pela média ou pela porcentagem. O método de Kaplan-Meier foi utilizado para calcular a estimativa da probabilidade de sobrevivência.

\section{Resultados}

Foram tratados 41 pacientes com AAA, sendo 36 homens e 5 mulheres. A média de idade foi de $68,8 \pm 7,1$ anos (variação: de 52 a 81). A morfologia foi predominantemente fusiforme, sendo apenas 2 casos $(4,8 \%)$ com a forma sacular. Entre os aneurismas fusiformes, o diâmetro variou de $5,2 \mathrm{a} 13 \mathrm{~cm}$, com média de $6,2 \mathrm{~cm}$. A artéria ilíaca comum estava acometida em 18 pacientes (43,9\%), 83,3\% das vezes unilateralmente. Os dados relacionados ao aneurisma estão resumidos na Tabela 3.

Houve necessidade de oclusão da artéria ilíaca interna em 15 pacientes (36,6\%), dos quais apenas 1 bilateral (por embolização em dois tempos). Desses 15 pacientes, 8 foram submetidos à embolização prévia e 1 à ligadura cirúrgica no ato do procedimento. Nos demais casos (40\%), a oclusão da artéria ilíaca interna ocorreu devido ao fato de a ancoragem distal da prótese ter sido realizada na artéria ilíaca externa.

A maioria dos pacientes foi submetida ao procedimento sob anestesia geral exclusiva $(73,2 \%)$. O bloqueio (ráqui ou

Tabela 2 - Critérios anatômicos de elegibilidade para tratamento endovascular

\begin{tabular}{lc}
\hline Colo proximal & $\geq 15 \mathrm{~mm}$ \\
Angulação do colo proximal & $<60^{\circ}$ \\
Diâmetro da artéria ilíaca externa & $\geq 7 \mathrm{~mm}$ \\
Presença de trombo ou calcificação & $>50 \% \mathrm{da}$ \\
extensa no colo proximal & circunferência \\
\hline
\end{tabular}

Tabela 3 - Dados referentes aos aneurismas

\begin{tabular}{lc} 
Diâmetro & $6,1(3,8-13) \mathrm{cm}$ \\
Morfologia & Número (\%) \\
Fusiforme & $39(95,1)$ \\
Sacular & $2(4,8)$ \\
Envolvimento da artéria ilíaca comum & \\
Unilateral & $15(36,6)$ \\
Bilateral & $3(7,3)$ \\
\hline
\end{tabular}

Tabela 4 - Dados referentes aos procedimentos

\begin{tabular}{lc}
\hline Tipo de anestesia & Número (\%) \\
\hline Geral & $30(73,2)$ \\
Ráqui & $6(14,6)$ \\
Peridural & $3(7,3)$ \\
Geral + ráqui & $2(4,9)$ \\
Tipo de prótese & \\
Bifurcada & $31(75,6)$ \\
Monoilíaca & $10(24,5)$ \\
Uso de extensões & \\
Proximal & $2(4,8)$ \\
Distal & $10(24,4)$ \\
Unilateral & $9(22,0)$ \\
Bilateral & $1(2,4)$ \\
Oclusão da artéria ilíaca interna & $15(36,6)$ \\
Marca da prótese & \\
Apolo (NANO endoluminal) & $17(41,5)$ \\
Excluder (Gore) & $16(39,1)$ \\
Talent" (Medtronic) & $4(9,8)$ \\
ELLA & $3(7,3)$ \\
Aorfix & $1(2,4)$ \\
\hline
\end{tabular}


peridural) foi utilizado 11 vezes, em 9 casos (22\%) como técnica única e em 2 casos $(4,8 \%)$ associado à anestesia geral.

O tempo cirúrgico médio foi de 193 minutos (de 90 a $375 \mathrm{~min}$ ). O principal modelo de prótese foi a bifurcada, utilizada em 75,6\% dos casos, sendo necessário uso de extensões em apenas 12 casos (29,2\%). Várias marcas de endopróteses foram empregadas. Essas marcas, assim como os dados supracitados, estão descritas na Tabela 4.

A taxa de sucesso técnico primário foi de $97,6 \%$, tendo sido necessária a utilização de uma extensão proximal para tratamento de vazamento tipo I em 1 paciente, sendo o sucesso técnico secundário $100 \%$.

A presença de vazamento intraoperatório (primário) ocorreu em cinco pacientes, sendo quatro do tipo II e 1 do tipo Ia, relatado no parágrafo anterior. Dos pacientes com vazamento do tipo II, apenas um não apresentou resolução do vazamento, com aumento do tamanho do saco aneurismático e necessidade de ligadura da artéria mesentérica inferior por videolaparoscopia durante o acompanhamento pós-operatório. Os dados referentes ao procedimento estão na Tabela 4.

Entre as complicações intraoperatórias, houve oclusão de artéria renal unilateral em um paciente, com tentativa de implante de stent sem sucesso. No seguimento pós-operatório, o paciente evoluiu com insuficiência renal crônica e necessidade de hemodiálise. Um paciente apresentou oclusão da artéria ilíaca externa direita e foi tratado com sucesso por meio de enxerto cruzado ilíaco-femoral com prótese de polietrafluor etileno expandido (PTFE).

O tempo médio de permanência em UTI foi de 2,88 dias (de 1 a 9 dias) e o tempo médio de internação foi de 7,5 dias (de 2 a 24 dias). Não havendo complicações, os pacientes foram alimentados no primeiro dia após a cirurgia. Não foram empregadas drogas antiplaquetárias no pós-operatório. As complicações pós-operatórias intra-hospitalares estão listadas na Tabela 5.

A mortalidade perioperatória foi de 4,8\% (2 pacientes Figura 1). Como causa do óbito tivemos dois pacientes com embolização visceral maciça (um no segundo e outro no terceiro dia de pós-operatório). Esses dois casos apresentavam trombos não somente no colo proximal, mas também na aorta suprarrenal.

Tabela 5 - Complicações intra-hospitalares

\begin{tabular}{lc}
\hline Complicação & Número de pacientes (\%) \\
\hline Isquemia intestinal & $2(4,8)$ \\
Hematoma de sítio cirúrgico & $1(2,4)$ \\
Pneumonia & $1(2,4)$ \\
Fibrilação atrial & $1(2,4)$ \\
Sangramento & $1(2,4)$ \\
\hline
\end{tabular}

No período de acompanhamento, 2 novos pacientes apresentaram vazamento tipo II provenientes de artéria lombar, sendo que esses pacientes não haviam apresentado vazamento diagnosticado no intraoperatório. No entanto, em nenhum desses pacientes houve aumento do saco aneurismático; portanto, estão em acompanhamento sem necessidade de intervenção. Houve necessidade de reintervenção em dois pacientes, sendo um deles relatado em parágrafo prévio referente a vazamento tipo II e ligadura da artéria mesentérica inferior (Figura 2). O outro paciente, após 26 meses do procedimento inicial, apresentou migração da prótese, tratado com implante de extensão proximal (Figura 3). Se considerarmos todos os pacientes que apresentaram vazamento, são obtidos oito casos (19,5\%), cinco

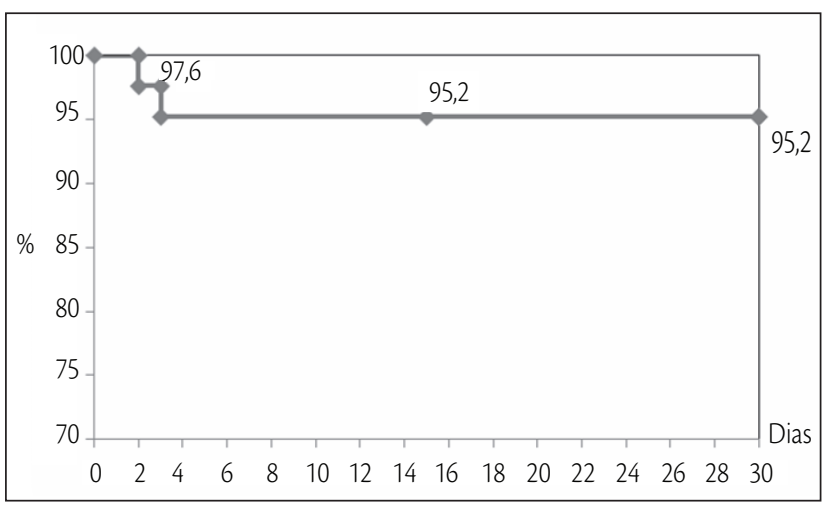

Figura 1 - Sobrevida perioperatória.

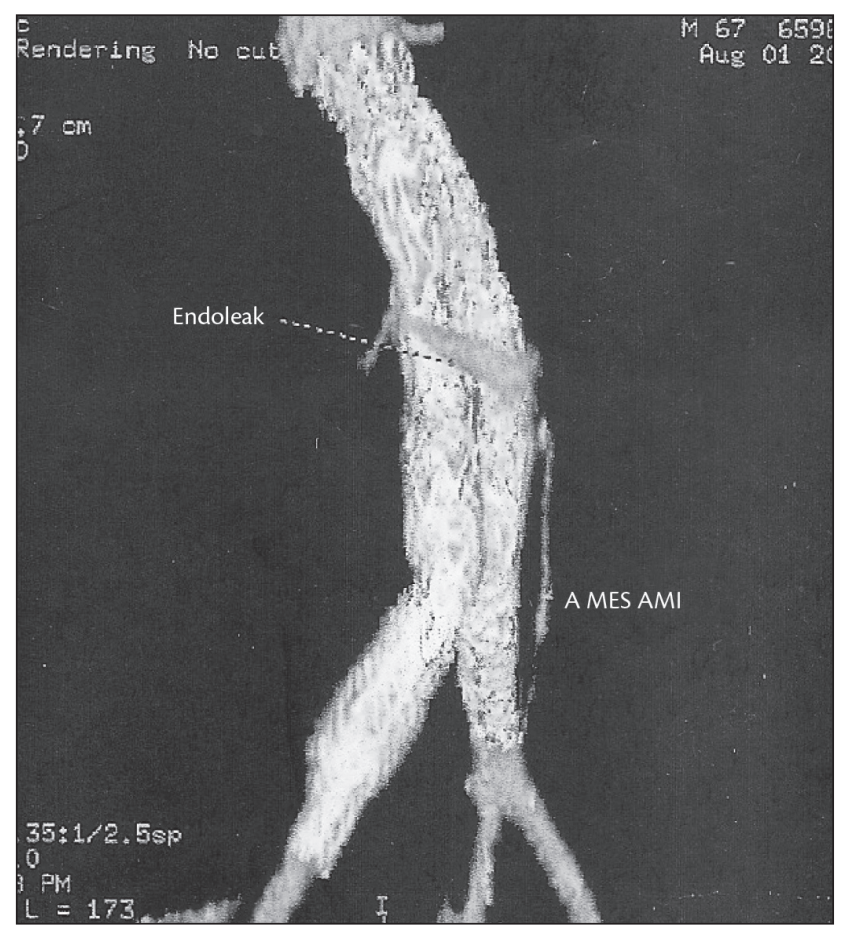

Figura 2 - Reconstrução de angiotromografia demonstrando endoleak tipo Il pela artéria mesentérica inferior (AMI). 
diagnosticados no intraoperatório (primários) e três no período de acompanhamento (secundários).

Com relação à mortalidade tardia, dois pacientes $(4,8 \%)$ apresentaram trombose de um dos ramos da prótese entre o segundo e o terceiro mês de acompanhamento e, apesar da instituição de tratamento cirúrgico, evoluíram com síndrome pós-revascularização grave e óbito. Posteriormente, nenhum outro paciente faleceu ou foi perdido o acompanhamento. A estimativa de probabilidade de sobrevivência aos 58 meses foi de 90,2\% (Figura 4).

O saco aneurismático apresentou redução de seu diâmetro em 21 casos (51,2\%), manteve-se estável em 18 casos $(43,9 \%)$ e apresentou crescimento em 2 , que foram submetidos à reintervenção.

\section{Discussão}

A ruptura do aneurisma de aorta abdominal é considerada uma verdadeira catástrofe abdominal, com taxa de mortalidade maior que $80 \%{ }^{1}$. Quando submetidos a tratamento cirúrgico aberto, os pacientes com aneurisma roto apresentam mortalidade de aproximadamente 50\%, taxa que não tem se alterado significantemente nos últimos anos $^{5}$. Pacientes com aneurisma medindo entre 5,5 e 5,9 cm de diâmetro transverso apresentam taxa anual de ruptura de $9,4 \%$, chegando a $32,5 \%$ quando o aneurisma atinge $7 \mathrm{~cm}^{6}$. Assim, para evitar esse desfecho, preconiza-se seu reparo de forma eletiva quando o diâmetro transverso do

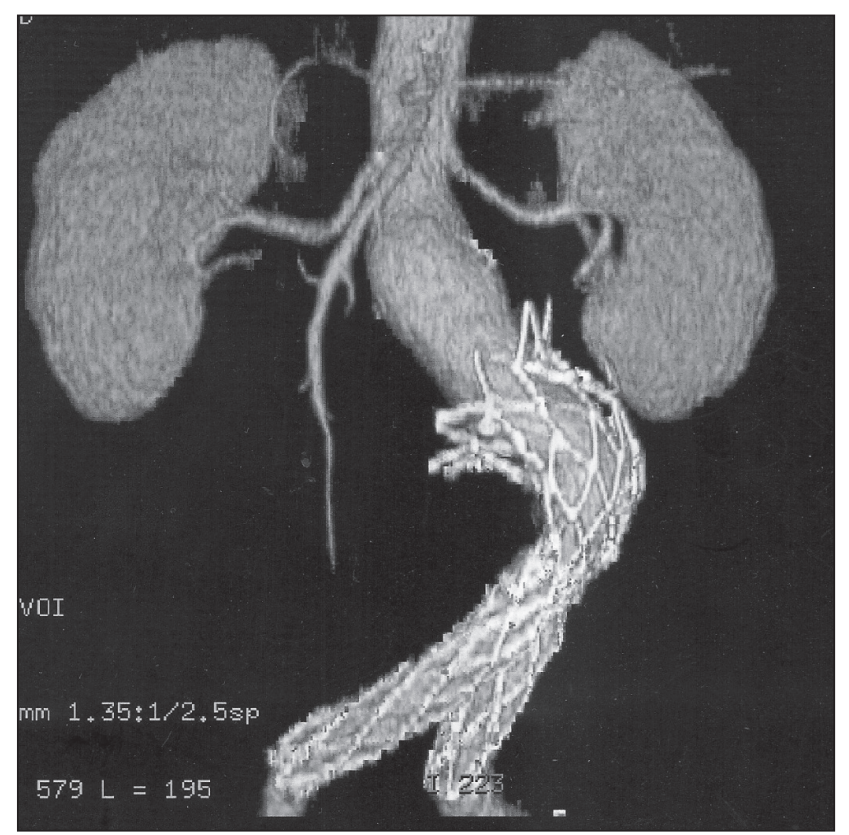

Figura 3 - Reconstrução de angiotomografia demonstrando migração da endoprótese. aneurisma fusiforme atinge medida de $5,5 \mathrm{~cm}$ para homens e 5,0 $\mathrm{cm}$ para mulheres ${ }^{1,7}$.

A eficácia do tratamento cirúrgico já é bem estabelecida, com taxas de falência do enxerto de apenas $0,3 \%$ ao $\mathrm{ano}^{2,3}$. No entanto, o tratamento convencional envolve recuperação prolongada e mortalidade perioperatória não desprezível $^{8}$, com taxas variáveis e que apresentam o valor médio de 6,8\% no Reino Unido9. Entre os autores brasileiros, encontramos resultados semelhantes com taxas de 3,3 a 5,3\% ${ }^{10-12}$. Em nosso serviço, a partir de outubro de 2004, quando iniciamos o tratamento do AAA por via endovascular, temos reservado a realização pela técnica convencional apenas para os casos em que a anatomia seja desfavorável ao procedimento e para pacientes com menos de 50 anos sem comorbidades proibitivas. Embora fosse interessante, os dados dos pacientes submetidos a tratamento convencional no mesmo período do estudo não foram compilados.

Devido à frequente associação de comorbidades nos pacientes portadores de aneurisma de aorta abdominal, é comum que um grupo desses pacientes seja considerado de risco cirúrgico elevado, ou mesmo proibitivo, para tratamento aberto.

O reparo endovascular foi desenvolvido como método menos invasivo alternativo ao reparo aberto do AAA. Semelhante a outros métodos minimamente invasivos, pacientes e médicos assumem esse benefício, mesmo antes que tenham sido comprovados seus resultados. Inicialmente, o reparo endovascular para o AAA era oferecido apenas para pacientes de alto risco. Estudos iniciais não randomizados mostraram menor morbidade operatória e menor tempo de internação para o reparo endovascular ${ }^{13-17}$. Apesar dessa redução na mortalidade também tem sido sugerida por esses estudos iniciais, isso ficou comprovado após a publicação do "EVAR-1"18. Esse estudo demonstrou uma redução de $65 \%$ na mortalidade perioperatória $(4,7 \%$ para o reparo

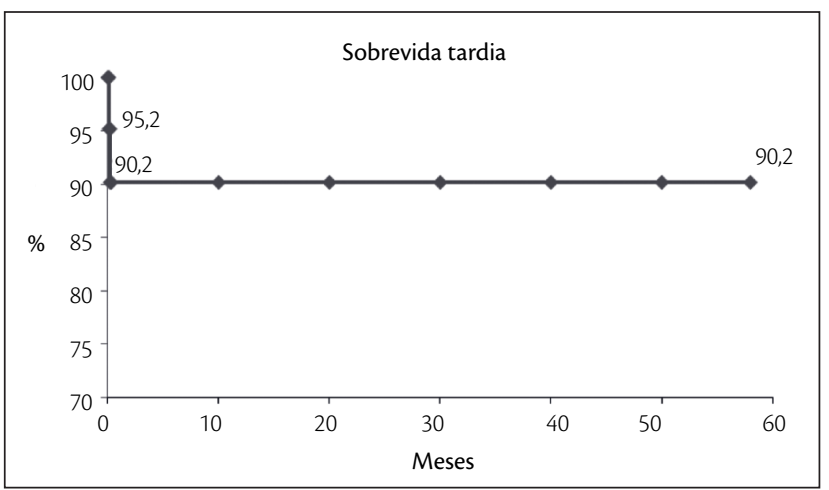

Figura 4 - Sobrevida tardia - estimativa de probabilidade de sobrevivência (método de Kaplan-Meier). 
aberto versus $1,7 \%$ para o endovascular). Ressalta-se que os pacientes desse estudo eram considerados elegíveis tanto para o tratamento aberto quanto para o tratamento endovascular, e não apenas pacientes de alto risco cirúrgico. Dois outros estudos controlados randomizados ${ }^{19,20}$ revelaram reduções semelhantes nas taxas de mortalidade perioperatória encontradas no estudo "EVAR-1", sendo que no estudo Dutch Randomized Endovascular Aneurysm Repair (DREAM) a taxa foi ainda menor com a técnica endovascular $\left(1,2\right.$ versus 4,6\% ${ }^{20}$. Em nosso meio, Mendonça et al. ${ }^{21}$ compararam o tratamento aberto com o endovascular para o AAA e anatomia favorável. A mortalidade encontrada foi de $6,45 \%$ para o tratamento aberto e de $5,55 \%$ para o endovascular, não sendo a diferença estatisticamente significativa. O mesmo autor, em descrição posterior ${ }^{22}$, relata taxa de mortalidade perioperatória de apenas 2,5\% em pacientes de alto risco cirúrgico (ASA III e IV), demonstrando a segurança do método mesmo nesse grupo de pacientes.

A mortalidade perioperatória de 4,8\% relatada em nosso estudo encontra-se acima desses estudos supracitados, porém totalmente compatível e aceitável como sendo o relato dos primeiros 41 casos operados, em que o efeito da curva de aprendizado na correção endovascular do AAA tem sido bem descrita ${ }^{23-25}$.

Os dois óbitos perioperatórios, que ocorreram no início de nossa série por embolização maciça em pacientes com trombos difusos, tanto no colo proximal como na aorta suprarrenal, despertaram-nos grande cautela na indicação da técnica nesses casos, apesar de esses pacientes apresentarem risco cirúrgico proibitivo para a correção aberta e seus aneurismas serem volumosos, com diâmetros transversos superiores a $7 \mathrm{~cm}$.

Os resultados iniciais favoráveis para pacientes de alto risco fizeram com que o reparo endovascular fosse oferecido para pacientes de baixo risco, desde que apresentassem critérios anatômicos favoráveis.

Apesar de esses dados terem sido atraentes do ponto de vista de morbimortalidade, houve preocupação a respeito da durabilidade dos materiais utilizados e, consequentemente, da necessidade de reintervenções com seus custos e riscos associados. Apesar de haver complicações tardias com o tratamento aberto, elas são menos comuns em relação ao tratamento endovascular. Isso fez com que alguns autores considerassem desnecessário o acompanhamento tardio para pacientes submetidos ao tratamento aberto ${ }^{2}$, o que já não é possível com a correção endovascular.

Desde a publicação original de Parodi ${ }^{4}$, tem ocorrido um rápido desenvolvimento tecnológico das endopróteses, tornando o procedimento mais seguro. Apesar da diminuição nas reintervenções com o desenvolvimento dos materiais, a base de registros Eurostar tem estimado taxa de reintervenções de $5 \%$ e taxa anual de ruptura de $1 \%{ }^{26,27}$. Consequentemente, tomografias e/ou ultrassonografias são necessárias para detectar vazamentos e possível migração das endopróteses. Essa migração, definida como um deslocamento caudal maior que $10 \mathrm{~mm}$, tem sido documentada em vários estudos e apresenta taxa média de ocorrência de $4 \%$, durante um acompanhamento superior a 12 meses $^{28}$. Em nosso levantamento, observamos apenas 1 caso $(2,4 \%)$, que necessitou do implante de uma extensão proximal com exclusão do aneurisma 26 meses após o procedimento inicial.

Uma complicação que não é inerente aos procedimentos endovasculares, mas que merece atenção, é a trombose de ramo do enxerto. Em nossa série, observamos essa complicação em 2 pacientes $(4,8 \%)$ entre o segundo e o terceiro mês de seguimento, e que evoluíram de forma insatisfatória com óbito por síndrome pós-revascularização grave, apesar da instituição de tratamento cirúrgico. Em revisão sistemática, feita por Drury et al. ${ }^{28}$, no levantamento de três estudos controlados randomizados, observou-se uma taxa maior dessa complicação $(6,4 \%)$ na correção endovascular quando comparado ao tratamento aberto (2,9\%). Uma possível explicação para essa maior taxa, seria, a nosso ver, o fato de que durante o tratamento cirúrgico convencional, o cirurgião elege o local preferencial com menos doença aterosclerótica para a realização da anastomose distal, o que não ocorre com as endopróteses que já vêm com medidas pré-estabelecidas, ancorando-se nem sempre em áreas ideais da artéria distal. Outra explicação seriam as angulações a que estão sujeitas as endopróteses que acompanham o trajeto natural das artérias muitas vezes acotoveladas ${ }^{29}$. Para reduzir esse tipo de complicação, Oshin et al. sugerem a utilização primária de stents não revestidos adicionais de uma maneira mais liberal em casos de acotovelamento ou estenose, seja do ramo da endoprótese ou da artéria receptora. A partir da adoção dessa tática não observaram nenhuma oclusão, tendo utilizado stents em 11,2\% dos casos operados $^{30}$.

Outra complicação que podemos observar quando necessitamos realizar a oclusão das artérias ilíacas internas bilateralmente ou de uma única artéria patente é a isquemia pélvica, com graus variados de manifestações clínicas, principalmente para a musculatura glútea e colón, além da disfunção sexual masculina. Em nossa série, realizamos a oclusão bilateral em apenas um caso, que não apresentou complicações. Cochennec et al. compararam a correção aberta com a endovascular em casos de AAA que envolviam a bifurcação ilíaca e observaram não haver diferença 
na mortalidade pós-operatória e nas complicações sistêmicas entre os dois grupos. Nos pacientes que foram submetidos ao reparo aberto, observaram maior número de complicações na parede abdominal e a tendência a uma maior frequência de isquemia colônica. Já no grupo endovascular, a claudicação glútea foi mais comum ${ }^{31}$.

$\mathrm{Na}$ tentativa de se preservar a perviedade das artérias ilíacas internas, endopróteses com ramos ilíacos bifurcados foram desenvolvidas ${ }^{32-34}$. Apesar da excelente taxa de sucesso técnico de 97,3\%, em 47 implantes, em 37 pacientes, observada por Ferreira et al. ${ }^{35}$, esse procedimento tem limitações anatômicas para seu emprego rotineiro. Karthikesalingam et al. quantificaram a proporção de pacientes com morfologia adequada para o uso desse tipo de material em estudo retrospectivo de 51 pacientes (66 aneurismas comprometendo a bifurcação ilíaca). Os autores obtiveram dois resultados: na primeira análise, seguindo as recomendações do fabricante da endoprótese, 38\% dos casos preenchiam os critérios exigidos. Na segunda análise, que considerou a opinião de cirurgiões experientes, apenas $29 \%$ seriam adequados para esse tipo de técnica. A causa mais comum de inadequabilidade foi a presença de uma artéria ilíaca interna aneurismática ${ }^{36}$.

Os vazamentos, caracterizados pela complicação exclusiva da correção endovascular, estiveram presentes em $19,5 \%$ dos casos em nossa série, sendo que em apenas 2 $(4,8 \%)$ houve necessidade de reintervenção. Desses dois casos, um se tratava de vazamento do tipo I e outro do tipo II.. Em revisão sistemática feita por Drury et al. ${ }^{28}$, ao analisarem os estudos randomizados controlados que verificaram a segurança e a eficácia do tratamento endovascular, observaram a presença de vazamento em $17,5 \%$ no período inferior a 30 dias, sendo $3,5 \%$ do tipo I e 14\% do tipo II. Já no acompanhamento superior a 12 meses, essa incidência elevou-se para $21,3 \%$, com ascensão do tipo I para $6,8 \%$, surgimento dos vazamentos tipo III $(4,2 \%)$ e redução do tipo II para $10,3 \%$. Nessa mesma revisão, os autores encontraram uma taxa de 16,2\% de intervenção secundária.

Apesar da necessidade de acompanhamento e de reintervenções, o seguimento de quatro anos mostrou que o reparo endovascular mantém uma taxa de mortalidade relacionada ao aneurisma $3 \%$ a menos que o reparo aberto ${ }^{18}$.

Acreditamos que a anatomia favorável seja o fator preponderante para o sucesso do procedimento. Os dados do Eurostar ${ }^{26}$ mostram que os melhores resultados foram obtidos em pacientes com aneurismas de diâmetro menor que $6,5 \mathrm{~cm}$ e anatomia favorável ao procedimento, independentemente do risco cirúrgico dos resultados, o que também foi observado por Mendonça et al. ${ }^{22}$.
O "EVAR-1"18 também mostrou que os melhores resultados foram conseguidos para pacientes com anatomia favorável. Além disso, os pacientes deste estudo foram considerados elegíveis tanto para o tratamento aberto quanto para o tratamento endovascular, ressaltando o benefício dos resultados com relação à anatomia favorável. Entre os dados a serem considerados na anatomia, o colo proximal favorável é o fator preponderante no sucesso dessa técnica. Um colo proximal com comprimento superior a $15 \mathrm{~mm}$, com pouca calcificação, trombos restritos a não mais que $50 \%$ de sua circunferência e com angulação inferior a $60^{\circ}$ é considerado o ideal ${ }^{29,37,38}$. A anatomia desfavorável do colo é a razão primária pela qual os pacientes com aneurisma aórtico infrarrenal têm que ser submetidos ao reparo aberto ${ }^{39}$.

Entretanto, com o aperfeiçoamento da técnica e o desenvolvimento de novos dispositivos, colos proximais com menos de $15 \mathrm{~mm}$ têm sido tratados. AbuRahma et al. ${ }^{40}$ analisaram uma série de 237 pacientes, sendo que 53\% deles apresentavam colo desfavorável sendo $24(10,1 \%)$ com 10 a $15 \mathrm{~mm}$ de extensão e $17(7,1 \%)$ com menos de $10 \mathrm{~mm}$.

$\mathrm{Na}$ análise da importância do comprimento do colo, verificaram um aumento na incidência de endoleak tipo I, tanto precoce como tardiamente, porém não houve aumento das reintervenções tardias, apenas das precoces com colocação de extensões proximais e stents expansíveis por balão. Entretanto, documentaram uma taxa mais elevada de complicações e mortalidade perioperatória em pacientes com colo proximal inferior a $15 \mathrm{~mm}$ de extensão. O aumento da taxa de mortalidade em 30 dias também foi observado por Leurs et al. ${ }^{41}$ no estudo Eurostar em pacientes com colo inferior a $15 \mathrm{~mm}$. Por outro lado, em recente publicação do estudo piloto da endoprótese Talent ${ }^{\oplus}$ com sistema de baixo perfil, colos tão curtos quanto $3 \mathrm{~mm}$ foram tratados, com taxa de mortalidade de 1,5\% em 30 dias. No acompanhamento por um período de cinco anos, a taxa de ruptura dos aneurismas foi de $3,5 \%$ e a conversão para cirurgia ocorreu em $0,9 \%(1 / 137)^{42}$.

Esses dados nos indicam a necessidade de se avaliar a realização do tratamento endovascular em pacientes com anatomia adversa. É provável que com a experiência crescente e com o desenvolvimento de novos materiais, os cirurgiões vasculares se sintam mais seguros em tratar esse grupo de pacientes, principalmente os que apresentarem risco cirúrgico elevado para o tratamento aberto.

Com o que observamos em nossa série, podemos concluir que, apesar do pequeno número de pacientes, os resultados obtidos parecem justificar a realização desse procedimento nos pacientes com anatomia adequada. 


\section{Agradecimento}

Ao Dr. Adamastor Humberto Pereira pelos ensinamentos recebidos durante a realização dos primeiros procedimentos.

\section{Referências}

1. Lederle FA, Wilson SE, Johnson GR, Reinke DB, Littooy FN, Acher CW, Ballard DJ, Messina LM, Gordon IL, Chute EP, Krupski WC, Busuttil SJ, Barone GW, Sparks S, Graham LM, Rapp JH, Makaroun MS, Moneta GL, Cambria RA, Makhoul RG, Eton D, Ansel HJ, Freischlag JA, Bandyk D; Aneyrism Detection and Management Affairs Cooperative Study Group. Immediate repair compared with surveillance of small abdominal aortic aneurysms. N Engl J Med. 2002;346:1437-44.

2. Hallett JW Jr, Marshall DM, Petterson TM, et al. Graft-related complications after abdominal aortic aneurysm repair: reassurance from a 36-year population-based experience. J Vasc Surg. 1997;25:277-84

3. Johnston KW. Nonruptured abdominal aortic aneurysm: six-year follow-up results from the multicenter prospective Canadian aneurysm study. Canadian Society for Vascular Surgery Aneurysm Study Group. J Vasc Surg. 1994;20:163-70.

4. Parodi JC, Palmaz J, Barone HD. Transfemoral intraluminal graft implantation for abdominal aortic aneurysms. Ann Vasc Surg. 1991;5:491-9.

5. Hoornweg LL, Storm-Versloot MN, Ubbink DT, Koelemay MJ, Legemate DA, Balm R. Meta analysis on mortality of ruptured abdominal aortic aneurysms. Eur J Vasc Endovasc Surg. 2008;35: 558-70.

6. Lederle FA, Johnson GR, Wilson SE et al. Rupture rate of large abdominal aortic aneurysms in patients refusing or unfit for elective repair. JAMA. 2002;287:2968-72.

7. United Kingdom Small Aneurysm Trial Participants. Long-term outcomes of immediate repair compared with surveillance of small abdominal aortic aneurysms. N Engl J Med. 2002;346: 1445-52.

8. Lee WA, Carter JW, Upchurch G, Seeger JM, Huber TS. Perioperative outcomes after open and endovascular repair of intact abdominal aortic aneurysms in the United States during 2001. J Vasc Surg. 2004;39:491-6.

9. The Vascular Society of Great Britain and Ireland. Fourth National Vascular Database Report 2004. Dendrite Clinical Systems, Oxfordshire 2005. ISBN 1-903968-12-7.

10. Becker M, Bonamigo TP, Faccini FP. Avaliação da mortalidade cirúrgica em aneurismas infra-renais da aorta abdominal. J Vasc Bras. 2002;1:15-21.

11. Menezes FH, Carchedi Luccas G, Matsui IA. Sobrevida tardia de pacientes submetidos à correção aberta eletiva de aneurisma de aorta abdominal. J Vasc Bras. 2007;6(3):218-224.

12. Carvalho ATY, Santos VP, Razuk Filho A, et al. Fatores de morbimortalidade na cirurgia eletiva do aneurisma da aorta abdominal infrarrenal: experiência de 134 casos. J Vasc Bras. 2008; 7:214-24.

13. Adriaensen ME, Bosch JL, Halpern EF, Myriam Hunink MG, Gazelle GS. Elective endovascular versus open surgical repair of abdominal aortic aneurysms: systematic review of short-term results. Radiology. 2002;224:739-47.

14. Bush RL, Johnson ML, Hedayati N, Henderson WG, Lin PH, Lumsden AB. Performance of endovascular aortic aneurysm repair in high-risk patients: results from the Veterans Affairs National Surgical Quality Improvement Program. J Vasc Surg. 2007;45: 227-33.

15. lannelli G, Monaco M Di Tomasso $L$ et al. Endovascular vs. open surgery of abdominal aortic aneurysm in high risk patients: a single center experience. Thorac Cardiovasc Surg. 2005;53:291-4.

16. Sukhija R, Aronow WS, Mathew J et al. Treatment of abdominal aortic aneurysms with an endovascular stent-graft prothesis in 96 high-risk patients. Cardiol Rev. 2005; 13:165-6.

17. Allaqaband S, Mortada ME, Tumuluri R, Kumar A, Bajwa TK. Endovascular stent graft repair of abdominal aortic aneurysm in high-risk patients: a single center experience. J Interv Cardiol. 2004;17:71-9.

18. Greenhalgh RM, Brown LC, Kwong GP, Powell JT, Thompson SG. Comparison of endovascular aneurysm repair with open repair in patients with abdominal aortic aneurysm (EVAR trial 1), 30-day operative mortality results: randomised controlled trial. Lancet. 2004;364:843-8.

19. Cuypers PWM, Gardien M, Buth J, Peels CH, Charbon JA, Hop WC. Randomized study comparing cardiac response in endovascular and open abdominal aortic aneurysm repair. Br J Surg. 2001;88:1059-65.

20. Prinssen $M$, Verhoeven EL, Buth J, et al. A randomised trial comparing conventional and endovascular repair of abdominal aortic aneurysms.("DREAM”) N Engl J Med 2004; 351: 1607-18.

21. Mendonça CT, Moreira RCR, Timi JRR, et al. Comparação entre os tratamentos aberto e endovascular dos aneurismas da aorta abdominal em pacientes de alto risco. J Vasc Br 2005;4(3):232-42

22. Mendonça CT, Moreira RCR, Carvalho CA et al. Tratamento endovascular de aneurismas da aorta abdominal em pacientes de alto risco cirúrgico. J Vasc Bras. 2009;8:56-64.

23. Ayerdi J, McLafferty RB, Markwell SJ, et al. Indications and outcomes of AneuRx phase III trial versus use of commercial AneurRx stent graft. J Vasc Surg. 2003;37: 739-43.

24. Howell MH, Zaqqa M, Villareal RP, Strickman NE, Krajcer Z. Endovascular exclusion of abdominal aortic aneurysms: initial experience with stent-grafts in cardiology practice. Tex Heart Inst J. 2000;27:136-45.

25. Ramaiah VG, Westerband A, Thompson C, et al. The AneuRx stent-graft since FDA approval: single-center experience of $230 \mathrm{ca}$ ses. J Endovasc Ther. 2002; 9:464-9.

26. Harris PL, Vallabhaneni SR, Desgranges P, et al. Incidence and risk factors of late rupture, conversion, and death after endovascular repair of infrarenal aortic aneurysms: the EUROSTAR experience. European Collaborators on Stent/graft techniques for aortic aneurysm repair. I Vasc Surg. 2000;32:739-49.

27. Harris PL, Buth J. An update on the important findings from the EUROSTAR EVAR registry. Vascular. 2004;12:33-8.

28. Drury D, Michaels JA, Jones L, Ayiku L. Systematic review of recent evidence for the safety and efficacy of elective endovascular repair in the management of infrarenal abdominal aortic aneurysm. $\mathrm{Br}$ J Surg 2005;92:937-46. 
29. von Ristow A, Pedron C, Gress MHT, Vescovi A, Massiére BV Aneurisma da aorta abdominal - tratamento pela técnica endovascular. In: Brito C). Cirurgia vascular - cirurgia endovascular Angiologia. Rio de Janeiro: Revinter; 2008. Cap 68. p. 1225-304.

30. Oshin OA, Fisher RK, Williams LA et al. Adjunctive iliac stents reduce the risk of stent-graft limb occlusion following endovascular aneurysm repair with the Zenith stent-graft. J Endovasc Ther. 2010;17:108-14

31. Cochennec F, Marzelle J, Alaire E, Desgranges P, Becquemin JP. Open vs endovascular repair of abdominal aortic aneurysm involving the iliac bifurcation. J Vasc Surg. 2010;51:1360-6.

32. Ziegler P, Avgerinos ED, Umscheid T, Perdikides T, Erz K, Stelter WJ. Branched iliac bifurcation: 6 years experience with endovascular preservation of internal iliac artery flow. J Vasc Surg. 2007;46:204-10.

33. Abraham CZ, Reilly LM, Schneider DB, et al. A modular multibranched system for endovascular repair of bilateral common iliac artery aneurysms. J Endovasc Ther. 2003;10:203-7.

34. Tielliu IF, Zeebregts CJ, van den Dungen JJ, Verhoeven EL. A modified technique for iliac artery branched endografting using a "tromboned" sheath. J Vasc Surg 2008;48:1605-8.

35. Ferreira M, Monteiro M, Lanziotti L. Technical aspects and midterm patency of iliac branched devices. J Vasc Surg. 2010;51545-50.

36. Karthikesalingam A, Hinchliffe RJ, Malkawi AH, et al. Morphological suitability of patients with aortoiliac aneurysms for endovascular preservation of the internal iliac artery using commercially available iliac branch graft devices. J Endovasc Ther. 2010;17:163-71.

37. Puech-Leão P. Correção endoluminal dos aneurismas. In: Maffei FHA. Doenças vasculares periféricas. $4^{a}$ ed. Rio de Janeiro: Guanabara Koogan Copyright;: 2008. Cap 93. p. 1386-97.

38. Hinchliffe RJ, Hopkinson BR. Natureza, frequência e fatores de risco dos procedimentos secundários realizados após a correção endovascular do aneurisma da aorta abdominal. In: Lobato AC. Tratamento endovascular das complicações aorto-ilíacas. Instituto de Cirurgia Vascular e Endovascular. São Paulo; 2008. Cap 17. p. 21522.

39. Dillavou ED, Muluk SC, Rhee RY, et al. Does hostile neck anatomy preclude successful endovascular aortic aneurysm repair? J Vasc Surg. 2003;38:657-63.

40. AbuRahma AF, Campbell J, Stone PA, et al. The correlation of aortic neck length to early and late outcomes in endovascular aneurysm repair patients. J Vasc Surg. 2009;50:738-48.

41. Leurs LJ, Kievit J, Dagniele PC, Nelemans PJ, Buth J; EUROSTAR collaborators. Influence of infrarenal neck length on outcome of endovascular abdominal aortic aneurysm repair. J Endovasc Ther. 2006;13:640-8.

42. Turnbull IC, Criado F), Sanchez L, et al. Five-year results for the Talent enhanced low profile system abdominal stent graft pivotal trial including early and long-term safety and efficacy. I Vasc Surg 2010;51:537-44.

Correspondência: José Manoel da Silva Silvestre Avenida Bandeirantes, 901, sala 504 - Vila Ipiranga CEP 86010-020 - Londrina (PR), Brasil Tel.: (43) 3339-6347 E-mail: silvestrejms@sercomtel.com.br

Contribuições dos autores: Concepção e desenho do estudo: JMSS Análise e interpretação dos dados: JMSS, FM, FT Coleta de dados: FM, GSS, ISP Redação do artigo: JMSS, FM Revisão crítica do texto: JMSS, WES, DMF Aprovação final do artigo: WES, DMF Análise estatística: JMSS

Responsabilidade geral pelo estudo: JMSS 